\title{
Il servizio è potere Un fatto biblico-evangelico e teologico
}

\author{
SILvANa FUŽINATO* \\ - https://doi.org/10.31823/d.28.2.3 • \\ UDC: $27-247.4$ - Original Scientific Paper
}

Received: $21^{\text {st }}$ September 2019 Accepted: $26^{\text {th }}$ May 2020

${ }^{*}$ Prof. Ass. Silvana

Fužinato, Facoltà di Teologia Cattolica di Đakovo, Università Josip Juraj Strossmayer di Osijek, Repubblica di Croazia, fuzinatosilvana@ gmail.com

Riassunto: Riflettendo sul servizio come modo di vivere il potere, è necessario ritornare alla Sacra Scrittura, specialmente al vangelo di Matteo che presenta Gesù come il Figlio dell'uomo a cui è dato ogni potere in cielo e in terra (cf. Mt 28, 16-20). Nella prima parte del nostro lavoro rifletteremo sul potere di Gesù di guarire, rivelato in modo particolare ed esplicito nelle opere del Messia (Mt 8 -9). Il potere della vita che viene dalla croce è il tema della seconda parte, dedicata al cammino del Figlio dell'uomo verso la croce (Mt 20, 17-28). Il potere come responsabilità è il terzo aspetto fondamentale del potere di Gesù, mostrato nell'giudizio finale del Figlio dell'uomo (Mt 25, 31-46). Il potere del Figlio dell'uomo manifestato nel potere di insegnare, di perdonare i peccati, di guarire e di scacciare $i$ demoni è il potere a servizio dell'uomo e della sua salvezza.

Parola chiave: potere, autorità, Figlio dell'uomo, servizio, salvezza, Matteo.

\section{Introduzione}

Riflettendo sul potere e sull'autorità della Chiesa, e sulla sua missione nel mondo di oggi, dobbiamo ritornare alle origini, cioè al Vangelo in cui Gesù ha rivelato il vero volto del potere e l'autentico senso del servizio cristiano. Riguardo a ciò, una delle pagine più significative è la conclusione del Vangelo di Matteo (28, 16-20). Infatti, esso conclude con le ultime parole di Gesù rivolte agli undici discepoli: $\gg$ Mi è stato dato ogni potere in cielo e in terra. Nell'andare, dunque, fate discepoli tutti i popoli, battezzandoli nel nome del Padre e del Figlio e dello Spirito santo, insegnan- 
do loro a osservare tutto ciò che vi ho comandato. Ed ecco, io sono con voi tutti i giorni, fino alla fine del mondo. $\ll$ Si tratta del Testamento del Risorto, di eccezionale importanza, in cui è pienamente rivelata l'identità del Messia, la sua missione e il mandato universale per i discepoli. ${ }^{1}$

All'inizio del versetto 18, il Risorto dichiara che gli è stato dato ogni potere in cielo e in terra. L'aoristo passivo $e d o t h \bar{e}-\gg \mathrm{mi}$ è stato dato $\ll$ va interpretato come passivo teologico $\mathrm{e}$ indica che Gesù partecipa in pienezza alla potestà universale, illimitata e assoluta di Dio, perché è il Figlio di Dio (cf. Mt 20, 19 e 11, 27). In altre parole, il passivo teologico da una parte sottende l'azione di Dio e dall'altra rimanda all'esercizio dell'autorità dopo la morte in croce. Gesù dunque, nella risurrezione, riceve la pienezza dell'autorità che verrà esercitata sulla totalità della creazione e nell'ambito divino. ${ }^{2}$ In questo modo il Figlio, che unico possiede la piena e universale exousia $\gg$ potenza $\ll$, di cui è stato reso partecipe da Dio, riceve la piena e definitiva ratificazione. ${ }^{3}$ Lo rivela anche l'atteggiamento dei discepoli, che vedendolo, si prostrarono davanti a lui (cf. Mt 20, 19) ${ }^{4}$ Riguardo all'identità del Messia è significativo notare che in questa ultima scena (Mt 28, 16-20) Gesù non viene chiamato con un grande titolo cristologico (Kyrios, Christos, Figlio dell'uomo), ma semplicemente col suo nome Gesù. In questo modo Matteo vuol sottolineare che Gesù che camminava lungo le strade della Palestina - ammaestrando i discepoli e le folle che lo seguivano, cacciando i demoni e guarendo i malati di ogni infermità e infine crocifisso - è il Figlio dell'uomo rivestito di ogni potere in cielo e in terra. ${ }^{5}$ Di quale potere si tratta?

L'uso del termine exousia in Mt 28, 19 è stato continuamente messo in relazione alla visione del Figlio dell'uomo contenuta in Dn 7, 13-14: »Guardando ancora nelle visioni notturne, ecco apparire, sulle nubi del cielo, uno simile ad un figlio di uomo; giunse fino al vegliardo e fu presentato a lui, che gli diede potere, gloria e regno; tutti i popoli, nazioni e lingue lo servivano; il suo potere è un potere eterno, che non tramonta mai, e il suo regno è tale che non sarà mai distrutto. $\ll \mathrm{Da}$ un breve paragone fra i due testi si possono dedurre diversi aspetti in comune. Prima di tutto il motivo del potere che in Daniele è dato al Figlio dell'uomo insieme alla gloria e al regno, mentre in Matteo è dato a Gesù. Il secondo aspetto in comune è che ambedue i testi rimandano a un mondo superiore a quello terrestre. Le nubi del cielo

${ }^{1}$ M. GRILLI, Scriba dell'Antico e del Nuovo. Il Vangelo di Matteo, Bologna, 2011, 109.

${ }^{2}$ S. GRASSO, Il Vangelo di Matteo. Commento esegetico e teologico, Roma, 2014, 832.

${ }^{3}$ J. LANGE, Das Erscheinen des Auferstandenen im Evangelium nach Matthäus. Eine traditions - und redaktionsgeschichtliche Untersuchung zu Mt 28, 16-20, Würzburg - Stuttgart, 1973, 177.

${ }^{4}$ Il verbo proskyneō - » prostrarsi $\ll$, »adorare «, nell'Antico Testamento indica la venerazione del vero Dio (cf. Gen 18, 2; 19, 1; Es 34, 8; Nm 22, 31; 2 Cr 7, 3; Is 27, 13; 49, 7).

${ }^{5}$ Cf. M. GRILLI, Scriba dell'Antico e del Nuovo, 112. 
in Daniele indicano la provenienza del Figlio dell'uomo e in Matteo si parla di un potere dato a Gesù in terra e in cielo. ${ }^{6}$ Però, si avvertono anche delle divergenze. La più significativa riguarda proprio il potere del Figlio dell'uomo. Mentre in Daniele $\gg$ il Figlio dell'uomo viene sulle nubi del cielo a giudicare, in Matteo non si parla di giudizio, ma di missione. Per l'evangelista il Figlio dell'uomo e il suo potere sono messi a servizio della salvezza. Gesù viene a salvare; il Signore esaltato che mediante la risurrezione appartiene pienamente all'exousia divina, perché è Dio che gli dà la potestà, viene a salvare tutti gli uomini. $\ll^{7}$ Inoltre, Matteo non descrive né la comparsa davanti al trono né la sottomissione dei popoli. A differenza di Matteo, in Daniele »l'exousia non è all'origine di un mandato missionario e 'i popoli' non sono convocati allo scopo di essere evangelizzati. $\ll^{8}$

La seconda domanda riguarda la natura dell'exousia attribuita a Gesù. Per coglierne gli aspetti dobbiamo ritornare al racconto evangelico precedente. Infatti, Gesù diverse volte aveva dimostrato il suo potere nell'insegnamento (Mt 7, 29; 8, 29; 21, 23.24.27), nel perdonare i peccati (Mt 9, 6.8), nello scacciare i demoni (Mt 10, 1), nell'intervento sui venditori del tempio ( Mt 21, 23), nei miracoli (cf. Mt 8, 9). ${ }^{9}$ Potremmo dire che tutta la vita di Gesù è una vita in cui viene esercitato il potere, ma si tratta di un potere per la vita dell'uomo, perché l'uomo viva la sua esistenza in pienezza. Ora cercheremo di individuare gli aspetti più significativi dell'exousia di Gesù che ci aiuteranno a cogliere pienamente il vero volto e il senso autentico del servizio a cui sono chiamati i discepoli di ogni tempo.

\section{Il potere di guarire}

Primo aspetto dell'exousia di Gesù si trova nei capp. 8 - 9 che presentano le opere del Messia. Dopo aver presentato Gesù come Messia che insegna con potere e autorità (Mt $5-7)$, ora il testo lo descrive come colui che guarisce le infermità del suo popolo. ${ }^{10} \mathrm{I}$ dieci miracoli che Gesù compie in questa sezione conosciuta come

${ }^{6}$ Cf. G. GHIBERTI, Le »grandi « apparizioni del Risorto, in: M. LÀCONI, ed., Vangeli Sinottici e Atti degli apostoli, Torino, ${ }^{2} 2008,271$.

${ }^{7}$ M. GRILLI, Scriba dell'Antico e del Nuovo, 113.

${ }^{8}$ M. GRILLI, Parola di Dio e linguaggio umano. Verso una pragmatica della comunicazione nei testi biblici, in: Gregorianum 94(2013)3, 540. Queste divergenze hanno condotto gli studiosi a cercare altri testi che potrebbero essere sullo sfondo di Mt 28, 16-20: la teofania in Es 19 - 20, la benedizione sacerdotale in $\mathrm{Nm}$ 6, 22-27, i mandati profetici e il decreto reale di Ciro in $2 \mathrm{Cr} 36,23$ (J. DANIEL - S. J. HARRINGTON, Il Vangelo di Matteo, Torino, 2005, 372).

${ }^{9}$ Cf. G. GHIBERTI, Le $\gg$ grandi $\ll$ apparizioni del Risorto, 271; A. J. LEVORATTI, Vangelo secondo san Matteo, in: ID. - E. TAMEZ - P. RICHARD (ed.), Nuovo Commentario Biblico: $i$ Vangeli, Roma, $2005,553$.

${ }^{10}$ S. GRASSO, Il Vangelo di Matteo, 252. 
la $\gg$ sezione dei miracoli « senza nessuna ombra di dubbio rivelano il potere di Gesù di guarire gli uomini dalle loro infermità. Vediamo ora la guarigione di alcuni personaggi paradigmatici in cui possiamo cogliere il senso del potere e dell'autorità di Gesù e dei suoi discepoli.

In Mt 8, 1-17 Gesù scende dal monte e guarisce un lebbroso sulla strada (8, 1-4), poi va in una città a Cafàrnao e guarisce il figlio ${ }^{11}$ di un pagano $(8,5-13)$ e infine entra nella casa di Pietro e guarisce una donna: la suocera $(8,14-15)$. Si tratta delle categorie più povere, più umili, più deboli, più indifese che nella Bibbia vengono chiamate gli 'anawim Jhwh - »i poveri di Jhwh $\ll$. »È bello questo andare di Gesù sulle strade degli uomini, nelle città ... con un intento liberante: sciogliere l'uomo dalle schiavitù, di cui le malattie sono un aspetto fondamentale. $\ll{ }^{12}$ E significativo notare che Gesù guarisce il lebbroso e la suocera di Pietro con il tocco della sua mano (cf. Mt 8, 3.15). Il tocco di Gesù stupisce ancora di più se teniamo conto del fatto che si tratta di un lebbroso che secondo Lev 13 - 14 era obbligato ad evitare il contatto con le altre persone. ${ }^{13}$ Per questo i lebbrosi ritenuti colpiti da Dio a causa di una malattia contagiosa la cui guarigione era paragonata alla risurrezione dei morti (cf. 2 Re 5, 7), erano esclusi dalla convivenza sociale e religiosa. ${ }^{14}$ Come dunque spiegare il tocco di Gesù? Il lebbroso non poteva essere guarito con la sola Parola?

Gli studiosi hanno dato diverse risposte. Alcuni fanno riferimento agli eventi dell'esodo, soprattutto a Es 7, in cui Dio dice a Mosè: »Gli Egiziani riconosceranno che io sono Jhwh, quando stenderò la mia mano contro l'Egitto e condurrò fuori di mezzo a loro i figli di Israele.« $(7,5) \cdot{ }^{15}$ Altri invece leggono il gesto alla luce del comportamento abituale del taumaturgo che in quel tempo si serviva di quel gesto per evidenziare la grandezza e il carattere magico. ${ }^{16}$ Secondo alcuni Padri della Chiesa il tocco di Gesù dimostra la sua superiorità sulla Legge che proibiva di toc-

${ }^{11}$ Il termine greco pais può significare sia $\gg$ servo $\ll$ sia $\gg$ figlio $\ll$. In questo caso riteniamo che si tratta del $\gg$ figlio « perché in 10, 24-25 Matteo per »servo « usa il termine doulos e in 17, 14-15 interscambia pais e hyios. Anche nel racconto giovanneo 4, 46-46 si tratta del $\gg$ figlio $\ll$ (hyios).

${ }^{12}$ M. GRILLI, Scriba dell'Antico e del Nuovo, 47.

${ }^{13}$ Secondo la Mishnah l'entrata del lebbroso in casa rende impuri perfino i mobili e le travi (Nega'im 13.11).

${ }^{14}$ Cf. S. GRASSO, Il Vangelo di Matteo, 253.

${ }^{15}$ Così per esempio R. PESCH, Il Vangelo di Marco, I, Brescia, 1980, 243-244. Però come giustamente afferma U. LUZ, Matteo, II, Brescia, 2010, 25, nota 3: »È improbabile, tanto per Matteo quanto per Marco (...), che si alluda ai miracoli di Mosè e di Aronne, in Egitto, dove non si stende la mano per toccare. $\ll$

${ }^{16}$ Cf. J. GNILKA, Il Vangelo di Matteo, I, Brescia, 2000, 437. 
care un lebbroso (cf. Lev 13, 45-46). ${ }^{17}$ Matteo userà la stessa espressione più avanti > per simboleggiare la potente protezione sotto la quale si trovano i discepoli di Gesù ${ }^{18}$ (cf. Mt 12, 49). Imposizione della mano è un gesto simbolico che esprime potenza e trasmissione della forza. Il gesto di distendere la mano e di toccare il lebbroso è dunque anzitutto l'espressione della divina potenza. ${ }^{19}$ Toccando l'intoccabile, cioè un uomo lebbroso, Gesù risana l'uomo colpito nella sua profonda fragilità, rendendo visibile da una parte la suprema volontà salvifica espressa nella Parola: »Lo voglio, sii sanato « (Mt 8, 3: Thelō, katharisthèti) e dall'altra la sua solidarietà con gli esclusi ed emarginati di cui il lebbroso è un esempio per molto tangibile. E infatti, poco più avanti Gesù mostrerà gli stessi sentimenti mangiando con i pubblicani e i peccatori (cf. Mt 9, 10-13).

Anche nella guarigione della suocera di Pietro (Mt 8, 14-15) il tocco di Gesù rappresenta la solidarietà con un uomo sofferente, cioè l'investimento della potenza messianica nel risanamento delle sofferenze umane. ${ }^{20} \mathrm{~A}$ differenza del racconto sui primi discepoli (Mt 4, 18.21) lo sguardo di Gesù nel nostro episodio non è elettivo, ma è uno sguardo di partecipazione al dolore della donna. Dal testo sembrerebbe che solo Gesù vede la donna che giaceva ammalata e che solo lui si accorge della sua sofferenza.

La potenza di guarire in modo esplicito è confermata nel sommario conclusivo sulle guarigioni di Gesù $(8,16-17)$ in cui Matteo afferma: »Venuta la sera, gli portarono molti indemoniati ed egli scacciò gli spiriti con la sua parola e guarì tutti i malati, perché si adempisse ciò che era stato detto per mezzo del profeta Isaia: Egli ha preso le nostre infermità e si è addossato le nostre malattie.« La ragione ultima per cui Gesù guarisce è dunque l'obbedienza e l'adempimento della Parola di Dio. Ė nella volontà di Dio che risiede il fondamento dell'autorità, inteso non come volontà di potenza, ma come volontà di servizio.

${ }^{17}$ GIOVANNI CRISOSTOMO, Omelie sul Vangelo di Matteo, 25, 2 afferma: »Perché, sanandolo con la volontà e la sua parola, ha aggiunto il gesto di toccarlo con la mano? A me sembra che non l'abbia fatto per nessun altro motivo, se non per dimostrare anche così che non era soggetto alla legge, ma al di sopra di essa.«, https://books.google.hr (25. 8. 2019.). Questa interpretazione non si addice bene alla visione di Matteo secondo la quale Gesù non solo obbedisce alla Legge (cf. 8, 4b), ma non intende abolirne neppure uno iota o un apice (cf. Mt 5, 17-18).

${ }^{18}$ U. LUZ, Matteo, II, 25. Nel vangelo di Matteo Gesù stende la mano anche per salvare Pietro che affonda nell'acqua (cf. Mt 14, 31).

${ }^{19}$ R. PESCH, Il vangelo di Marco, Brescia, 1980, 243-244.

${ }^{20}$ S. GRASSO, Il Vangelo di Matteo, 267, afferma che in questo caso, come pure in Mt 8, 3, »il verbo aptomai più che un gesto drammatico o enfatico del taumaturgo, segnala il contatto volutamente fisico da parte di Gesù con l'ammalata.« 
Matteo prende la citazione dal quarto canto del Servo sofferente di Isaia $(53,4)$. Però si tratta di una traduzione matteana più vicina al testo ebraico che non alla traduzione greca della LXX. ${ }^{21}$ Infatti, il testo ebraico parla del servo che $\gg$ si è caricato delle nostre infermità e si è addossato i nostri dolori $\ll$. La LXX sostituisce il termine $\gg$ infermità $\ll$ con quello dei $\gg$ peccati $\ll$. Matteo si concentra invece non sui peccati, ma sulle malattie.

È significativo notare che $\gg$ farsi carico $<$ e $\gg$ portare i pesi $\ll$ non si adatta bene al contesto di Mt $8-9$, perché in essi, soprattutto in 8, 1-17, a differenza di Isaia 53, 3-5, Matteo non presenta il servo schiacciato sotto il peso delle debolezze umane. Gesù non prende su di sé le malattie, ma piuttosto le elimina con la sua potenza. Da questo si deduce che Matteo ha utilizzato la citazione di Is 53 piuttosto liberamente, applicandola non alla passione, ma alle guarigioni miracolose. In base al contesto, dove si parla della potenza di Gesù di guarire, cioè della sovranità che risana, i verbi lambanō e bastazō non hanno il significato di $\gg$ portare $\ll, ~ \gg$ prendere un carico $\ll$, ma di $\gg$ togliere $\ll$ e $\gg$ portar via « (cf. Mt 3, 11; Gv 12, 6). In questo modo Matteo vede in Gesù il messia di Israele, che con la sua potenza sovrana, elimina le fragilità degli uomini. ${ }^{22}$ In fine insieme a S. Grasso possiamo concludere che »questa trasformazione matteana, suscitata sia dal contesto evangelico, sia dall'intervento dell'autore sulla traduzione, serve così a descrivere l'atteggiamento solidale di Gesù, il messia che viene in soccorso dei bisognosi e degli ammalati attraverso i suoi gesti potenti. $\ll^{23}$

Analogamente al Servo sofferente di Isaia, Matteo presenta Gesù messia, solidale con l'uomo sofferente. Proprio grazie alla sua solidarietà con le debolezze umane Gesù guarisce e salva i miseri reintegrandoli pienamente nella pienezza della vita sociale e religiosa e nella loro dignità di figli di Dio. L'operare miracoloso di Gesù è dunque l'espressione della sua autorità che è al servizio dell'uomo e della sua salvezza. La potenza di Gesù di guarire è il segno supremo della sua solidarietà con gli umili e gli esclusi: lebbrosi, pagani, donne, a cui Gesù restituisce la dignità, reintegrandoli pienamente nel nuovo popolo messianico ristabilito nella salute e nella pace. Questa autorità e potenza di guarigione si può capire solo alla luce della croce: il segno supremo della potenza di Gesù intesa come servizio all'uomo. In essa troviamo un altro fondamentale aspetto della potenza di Gesù.

\section{Il potere della vita che viene dalla croce}

La morte di Gesù è il segno supremo del potere del Figlio dell'uomo vissuto nella totale donazione di sé stesso. In Mt 20, 17-19 l'autore presenta il cammino del

\footnotetext{
${ }^{21}$ A. MELLO, Evangelo secondo Matteo, Magnano, 1995, 153.

${ }^{22}$ U. LUZ, Matteo, II, 36.

${ }^{23}$ S. GRASSO, Il Vangelo di Matteo, 269-270.
} 
Figlio dell'uomo verso la croce. Mentre saliva a Gerusalemme Gesù prende in disparte i dodici e per la terza volta annuncia la passione che comprende la consegna ai sacerdoti e agli scribi, la condanna a morte, la consegna ai pagani, i dileggi, gli sputi, la flagellazione, l'uccisione e la risurrezione. Di fronte a tale annuncio Matteo riporta la domanda della madre di Giacomo e di Giovanni, i figli di Zebedèo: ${ }^{24}$ $\gg$ Di' che questi miei figli siedano uno alla tua destra e uno alla tua sinistra nel tuo regno.« (Mt 20, 21). ${ }^{25}$ Alla sorprendente contro-domanda di Gesù: »Potete bere il calice che io sto a bere? « i due discepoli rispondono direttamente: $\gg$ Lo possiamo $\ll($ Mt 20, 22). La metafora del $\gg$ calice $\ll$ nei profeti, in particolare in Geremia (Ger 25, 15; 49, 12; Lam 4, 21), indicava la coppa dell'ira divina, cioè il giudizio di Dio, ma nel giudaismo più tardo indicava il destino che tocca ad ogni uomo e, in senso più specifico, la morte. ${ }^{26}$ Ovviamente, in questo caso, come si deduce dal contesto dell'annuncio della passione, si tratta del calice della sofferenza e della morte. Udendo ciò, gli altri dieci discepoli si sdegnarono contro i due, nonostante Gesù avesse respinto la loro domanda. Riguardo alla loro reazione, U. Luz si chiede se, con la loro gelosia, gli altri discepoli non dimostrano di essere fatti della stessa pasta dei figli di Zebedèo e risponde: $\gg$ La voglia di esser grandi sulla terra $\mathrm{o}$, per compensazione religiosa, nell'aldilà, evidentemente non è una caratteristica solo di alcuni, ma di tutti i discepoli di Gesù. $\ll{ }^{27}$

Dopo aver ricordato, con ironia e forti tinte negative, l'atteggiamento e il comportamento delle autorità ufficiali di questo mondo (cf. Mt 20,25), Gesù presenta un altro modo di vivere le relazioni nella Chiesa e l'atteggiamento fondamentale della comunità dei discepoli, dove vige un nuovo ordine: $\gg$ Non così dovrà essere tra di voi; ma colui che vorrà diventare grande tra voi, si farà vostro servo, e colui che vorrà essere il primo tra voi si farà vostro schiavo; appunto come il Figlio dell'uomo, che non è venuto per essere servito, ma per servire e dare la sua vita in riscatto per molti.« (Mt 20, 26-28). ${ }^{28}$ Rovesciando la logica di questo mondo e il sistema di potere che vige nei rapporti umani, Gesù pone davanti ai suoi discepoli un altro modo di vivere l'autorità: il servizio. Uno solo è il Maestro (cf. Mt 23, 10-11) e ai discepoli compete il ministero della diakonia e della doulia - »servizio «. In entrambi

\footnotetext{
${ }^{24}$ Questi due fratelli il lettore ha incontrato in Mt 4, 21-22 nella scena della loro vocazione. Inoltre erano presenti alla trasfigurazione insieme a Pietro $(\mathrm{Mt} 17,1)$ e saranno i più vicini a Gesù in Getsemani (Mt 26, 37).

${ }^{25}$ In Marco i due discepoli si rivolgono personalmente a Gesù (cf. 10, 35-37).

${ }^{26}$ A. MELLO, Evangelo secondo Matteo, 453; U. LUZ, Matteo, III, Brescia, 2013, 213.

${ }^{27}$ U. LUZ, Matteo, III, 213.

${ }^{28}$ M. GRILLI, L'impotenza che salva. Il mistero della croce in Mc 8,27 - 10,52. Lettura in chiave comunicativa, Bologna, 2009, 137.
} 
i casi le due figure rappresentano l'antipotere e l'antiprestigio. ${ }^{29}$ Però, come osserva bene U. Luz:

$\gg$ Non si tratta di indicare una via alla grandezza nuova, più nobile di quella del dominio e del potere, bensì di rimuovere totalmente la volontà di essere grandi, perché basta il pensiero più sottile della propria volontà di essere grandi per rovinare il servizio genuino. Perciò Gesù sceglie qui di esprimersi per paradossi: 'Chi vuol essere grande' deve diventare piccolo e liberarsi del pensiero della grandezza. $\ll^{30}$

Unica via da seguire per diventare piccoli e ultimi è la via del servizio dimostrata chiaramente nella vita del Figlio dell'uomo $\gg$ che non è venuto per essere servito, ma per servire e dare la sua vita in riscatto per molti $\ll$ (Mt 20,28). Cosa significa per Gesù diakoneō - »servire « si deduce dall'espressione che segue dounai tēn psychèn - »dare la vita «. In Sir 29, 15 essa significa »mettere a repentaglio la vita « e in $2 \mathrm{Mac} 7,37$ indica il sacrificio della vita da parte del martire. Il servizio dunque è inteso nella sua forma radicale e fondamentale del sacrificio della vita. ${ }^{31}$ Contro le forme istintive e perverse di personalismo e arrivismo, di abuso e prevaricazione che giacevano nel cuore dei suoi discepoli, Gesù dice radicalmente: »Non sarà così tra voi $\ll(M t 20,26)$, presentando il Figlio dell'uomo, ${ }^{32}$ messia deriso e crocifisso che dona la vita, come unico modello per qualsiasi ruolo o funzione ecclesiale da vivere. ${ }^{33}$ Più volte, nel Vangelo, Gesù aveva detto ai suoi discepoli che la sequela concerne la rinuncia alla propria vita. Infatti, in Mt 10, 38-39 Gesù invita a prendere la sua croce e a seguirlo dichiarando: $\gg$ Chi avrà trovato la sua vita, la perderà: e chi avrà perduto la sua vita per causa mia, la troverà.« Più avanti in Mt 16, 24-25 dopo il primo annuncio della passione Gesù dirà ai suoi discepoli: »Se qualcuno vuol venire dietro a me rinneghi se stesso, prenda la sua croce e mi segua. Perché chi vorrà salvare la propria vita, la perderà; ma chi perderà la propria vita per causa mia, la troverà.« Però, a questo punto ci poniamo la domanda fondamentale: $\gg$ Per chi dare la propria vita? E per quale motivo? « La risposta ci è offerta da un'espressione singolare che si trova nel testo: lytron anti pollōn - »in riscatto per molti $\ll$.

Nei sinottici il termine lytron, che corrisponde all'ebraico kōfēr, appare solo qui e in Mc 10, 45 e significa $\gg$ prezzo del riscatto $\ll$. Nel greco profano l'espressione indica il prezzo per affrancare gli schiavi e, nella LXX, il risarcimento pecuniario espiato-

\footnotetext{
${ }^{29}$ S. GRASSO, Il Vangelo di Matteo, 598.

${ }^{30}$ U. LUZ, Matteo, III, 214.

${ }^{31}$ M. GRILLI, L'impotenza che salva, 137.

32 »'Il Figlio dell'uomo' è una denominazione che Gesù stesso si è dato e che include tutta la sua opera, la sua attività terrena, la sua morte e la sua esaltazione futura.« (U. LUZ, Matteo, III, 215-216.)

${ }^{33}$ S. GRASSO, Il Vangelo di Matteo, 598.
} 
rio sostitutivo della pena di morte (Es 21, 30). ${ }^{34}$ Alcuni autori ${ }^{35}$ leggono la morte di Gesù alla luce della morte del Servo del Signore in Is 53, 11-12: $\gg$ Dopo il suo intimo tormento vedrà la luce e si sazierà della sua conoscenza; il giusto mio servo giustificherà molti, egli si addosserà le loro iniquità. Perciò io gli darò in premio le moltitudini, dei potenti egli farà bottino, perché ha spogliato se stesso fino alla morte ed è stato annoverato fra gli empi, mentre egli portava il peccato di molti e intercedeva per i colpevoli.« Riguardo a ciò ci sembrano appropriate le osservazioni di M. Grilli che a proposito del testo parallelo in Mc 10, 45 conclude:

$\gg$ Nel nostro testo non è l'idea della transizione o di un sacrificio riparatore che viene alla ribalta, come se la morte di Gesù fosse un prezzo da pagare a Dio, o peggio ancora, a Satana, per la liberazione degli uomini. Gesù non è il 'capro espiatorio', che si sostituisce all'umanità peccatrice prendendo la colpa su di sé e pagando così, con la croce, il prezzo dovuto a Dio a causa del peccato. Questa idea commerciale della salvezza, ancora diffusa, va eliminata, perché estranea alla letteratura biblica. La metafora contenuta in lytron anti pollon evoca solo l'idea del costo che appartiene a ogni dono della vita. Dare la vita costa, ed è questo che Dio ha pagato per amore dell'uomo (...) Con tale espressione, dunque, i lettori riconoscono che nel servizio radicale di Gesù, espresso nel dono della propria esistenza, Dio ha stabilito la liberazione definitiva dell'uomo. Gesù, il Figlio dell'uomo, accetta liberamente la passione, per amore. $\ll^{36}$

Alla luce di quanto detto, possiamo concludere che l'exousia di Gesù si è pienamente rivelata nella sua morte, che è il segno supremo dell'amore per l'uomo e la sua salvezza. Nell'amore totale, gratuito e universale del Figlio dell'uomo; per ogni uomo e ogni donna anche l'autorità e il servizio dei discepoli di ogni tempo trovano il suo vero senso: vivere per qualcuno / Qualcuno. Il vero potere non è quello che si serve dell'altro, ma quello che serve l'altro; non è quello che possiede l'altro, ma quello che gli appartiene; non è il potere di padronanza che utilizza l'altro come strumento per i propri interessi, ma quello che dona sé stesso. La perfezione del potere come servizio consiste nel »vivere per l'altro « come il Figlio dell'uomo, nell'amore e nel dono della propria vita. Alla luce del ministero di Gesù, e nel Nuovo testamento, servire esprime la concretezza dell'amore. ${ }^{37}$

${ }^{34}$ U. LUZ, Matteo, III, 216.

${ }^{35}$ Per esempio A. MELLO, Evangelo secondo Matteo, 356; G. MICHELINI, Matteo, 324-325.

${ }^{36}$ M. GRILLI, L'impotenza che salva, 138. Anche U. LUZ, Matteo, III, 216, sostiene che Is 53, 10-12 non ha nessun aggancio terminologico con il nostro testo. Anzi secondo Luz esso ne anche altrove non ha per Matteo nessun significato particolare.

${ }^{37}$ S. FAUSTI, Una comunità legge il Vangelo di Matteo, Bologna, 2007, 397. 
Ai figli di Zebedèo che cercavano i primi posti nel suo Regno, Gesù indica la via del dono di sé come unica via del potere, capovolgendo tutte le strutture del potere umano. »In questo modo egli dà alla chiesa un orientamento fondamentale, precisamente verso il basso, sul servizio. Si tratta, in ultima analisi, dell'orientamento verso il suo cammino, il cammino del figlio dell'uomo. Per Matteo la struttura di servizio della chiesa significa la rinuncia a qualsiasi struttura di dominio nella chiesa. $\ll^{38}$

\section{Il potere come responsabilità}

Vorrei toccare per ultimo l'argomento del potere come responsabilità desumendo il tema da una pagina famosa e gloriosa di Matteo che parla del momento ultimo per il mondo e per tutti noi. Si tratta del giudizio finale raffigurato in $\mathrm{Mt}$ 25, 31-46, dove anche se non appare il termine exousia, in esso Gesù viene descritto come il Figlio dell'uomo veniente sopra le nubi del cielo, con grande potenza e gloria (cf. Mt 24, 30; 25, 31; 16, 27; 19, 28). L'autorità e il potere del Figlio dell'uomo in questo caso vengono dunque indicati dal suo stare seduto sul trono, il segno del potere di Dio su tutta la terra. ${ }^{39}$ Anche qui la figura del Figlio dell'uomo che viene sulle nubi del cielo è ripresa da $\mathrm{Dn} 7,13$, mentre l'accompagnamento da tutti i suoi angeli richiama Zc 14, 5, e altri testi apocalittici giudei, come per esempio il Libro delle parabole di Enoch. ${ }^{40}$ Come giustamente osserva una gran parte degli studiosi, ${ }^{41}$ in Mt 25, 31-46 il Figlio dell'uomo è il giudice escatologico.

Davanti al regno del Figlio dell'uomo saranno radunate panta ta ethne - >tutte le genti $\ll$. Di quale genti si tratta? Il termine goyîm nell'Antico Testamento indicava i popoli pagani. Anche nella LXX il termine ta ethnè è usato in riferimento ai popoli stranieri.$^{22}$ Con ogni probabilità, comunque, qui si tratta di tutti i popoli, inclusa la comunità dei credenti. ${ }^{43}$ Infatti, in Mt $24-25$ possiamo facilmente riconoscere il motivo dall'Antico Testamento e dalla letteratura apocalittica: raduno escatologico

\footnotetext{
${ }^{38}$ U. LUZ, Matteo, III, 217.

${ }^{39}$ Cf. Sal 11, 4; 47, 9; 93, 2; 103, 19; Ger 3, 17; Dn 7, 9 (S. GRASSO, Il Vangelo di Matteo, 718).

${ }^{40}$ G. MICHELINI, Matteo. Introduzione, traduzione e commento, Cinisello Balsamo, 2013, 399.

${ }^{41}$ U. LUZ, Matteo, III, 650; G. MICHELINI, Matteo, 399-400; A. MELLO, Evangelo secondo Matteo, 437.

${ }^{42}$ Cf. Gen 18, 18; Dt 23, 27; Is 2, 2; 40, 17; 52, 10; Ger 3, 17.

${ }^{43}$ Cf. U. LUZ, Matteo, III, 651; S. GRASSO, Il Vangelo di Matteo, 718; W. TRILLING, Das wahre Israel. Studien zur Theologie des Matthäus-Evangeliums, Leipzig, ${ }^{3} 1975,26-27$. S. FAUSTI, Una comunità legge il Vangelo di Matteo, 503; J. P. HEIL, Final Parables in the Eschatological Discourse in Matthew 24 - 25, in: W. CARTER, J. P. HEIL, Matthew's Parables. Audienced-oriented Perspectives, Washington, 1997, 201.
} 
di tutti i popoli della terra. ${ }^{44}$ Matteo, dunque, presenta un giudizio finale universale del mondo e della Chiesa, che riguarda tutti. In Mt 25, 31-46 infatti non troviamo nessun segnale, né grammaticale né sintattico, che indicherebbe un cambiamento di interlocutori rispetto ai discepoli menzionati nel testo che precede. Il giudizio di cui parla Matteo è per tutti gli uomini e, dunque, anche per la Chiesa. ${ }^{45}$

La seconda domanda riguarda il metro del giudizio. Esso non è il rapporto con una qualsiasi legge formalmente cristiana, ma con le opere di misericordia verso $\mathrm{i}$ più bisognosi della terra: affamati, assetati, forestieri, nudi, malati, carcerati, i quali sono quasi un $\gg$ sacramento $\ll$ della presenza storica del Figlio dell'uomo. ${ }^{46}$ Ovviamente, si tratta di una semplificazione delle opere, ma è proprio l'adempimento di esse il metro del giudizio. Un analogo programma troviamo anche nel giudaismo. Così in Sota 14a, Rabbi Chama' bar Chanina' commenta Dt 13, $5 \gg$ Voi seguirete il Signore vostro Dio « in questo modo:

$\gg$ Come Dio ha vestito quelli che erano nudi (Abramo ed Eva), vesti anche tu quelli che sono nudi; come Dio ha visitato gli ammalati (Abramo), tu pure visita gli ammalati; come Dio ha consolato gli afflitti (Isacco), consola anche tu gli afflitti; come Dio ha seppellito i morti (Mosè), tu pure seppellisci i morti. $\ll{ }^{47}$

Dunque, anche se le opere di solidarietà si trovano nella tradizione biblica e giudaica e soprattutto in quella profetico-sapienziale, è necessario accentuare un tratto decisamente matteano: in nessun altra religione il giudice si identifica con $\mathrm{i} \gg$ fratelli più piccoli « (cf. vv. 40.45) per cui il destino eterno di un individuo si decide sulla base dell'atteggiamento tenuto verso di loro. ${ }^{48} \mathrm{Chi}$ sono $\mathrm{i} \gg$ fratelli più piccoli $\ll$

${ }^{44}$ Cf. Is 2, 1-5; 25, 6-8; 66, 18b-24; Ez 34, 12-23, Dn 7, 14; Gl 4, 1-3; Mi 4, 1-8; Sof 3, 16-20.

${ }^{45}$ In favore a questa interpretazione U. LUZ, Matteo, III, 651-652 riporta i seguenti argomenti: a) Tutta quanta la parenesi di 24, $32-25,30$ non servirebbe a niente se non terminasse con la rappresentazione di un giudizio nel quale è coinvolta la comunità; b) un giudizio separato, nel quale il Figlio dell'uomo giudica solamente i non cristiani, sarebbe in totale contraddizione con la concezione matteana della chiesa; c) Matteo aveva chiuso tre dei suoi grandi discorsi precedenti con testi che parlavano del giudizio che avrebbe riguardato anche la comunità. Non è verosimile che in questo caso Matteo abbandonerebbe lo schema costante parlando di un giudizio che riguarda gli altri, i non cristiani; d) entrambi i gruppi apparsi in giudizio si rivolgono al giudice chiamandolo kyrie, appellativo che chiaramente appartiene al vocabolario della comunità. Proprio mediante tale appellativo i lettori vedranno confermata la loro identificazione con i due gruppi alla destra e alla sinistra del figlio dell'uomo.

${ }^{46}$ A. MELLO, Evangelo secondo Matteo, 438; M. GRILLI, Il discorso della montagna. Utopia o prassi quotidiana?, Bologna, 2016, 75.

${ }^{47}$ Preso da A. MELLO, Evangelo secondo Matteo, 438.

${ }^{48} \mathrm{M}$. GRILLI, Il discorso della montagna, 75. 
del Figlio dell'uomo? Alcuni autori vi hanno visto i discepoli di Gesù, cioè i primi predicatori, ${ }^{49}$ altri i poveri delle comunità cristiane ${ }^{50}$ o i poveri in generale, ${ }^{51}$ altri i cristiani ${ }^{52}$ o i giudeo-cristiani, o ancora i missionari giudei, i testimoni. ${ }^{53}$ Matteo parla dei $\gg$ piccoli $\ll$ anche in 10, 40-42 e 18, 6. A differenza di questi due testi però, in Mt 25 »i più piccoli « sono qualificati semplicemente per uno stato di povertà oggettiva e non per una appartenenza ecclesiale. Non si tratta solo di quelli che credono in Gesù, ma di uomini di cui i discepoli devono assumere la responsabilità.

Infatti, l'identificazione del Figlio dell'uomo con $\gg$ i più piccoli $\ll$ si comprende pienamente alla luce dei versetti seguenti che iniziano il racconto della passione: $\gg$ Terminati tutti questi discorsi, Gesù disse ai suoi discepoli: 'Voi sapete che fra due giorni è la Pasqua e il Figlio dell'uomo sarà consegnato per essere crocifisso'.« (Mt 26, 1-2). A. Fumagalli ritiene che questi versetti sono un commento alla pericope precedente, che si era conclusa lasciando un interrogativo in sospeso: $\gg$ Chi sono $i$ piccoli? $\ll{ }^{54} \mathrm{Ai}$ discepoli che vogliono conoscere i segni della fine e della venuta del Figlio dell'uomo (cf. Mt 24,3) Gesù rivela una nuova percezione della sua presenza nella storia. Nel tempo dell'attesa che precede il ritorno glorioso di Cristo, il Figlio dell'uomo è il crocifisso (cf. Mt 28, 5) e lo si incontra nei crocifissi: affamati, assetati, stranieri, carcerati ... Il Figlio dell'uomo potente e glorioso è diventato uno di loro. Infatti, più tardi, quando durante la passione i soldati del governatore lo spoglieranno mettendogli addosso un manto scarlatto, una corona di spine sulla testa e nella destra una canna, e inginocchiandosi davanti a lui lo scherniranno dicendo: $\gg$ Salve, re dei Giudei!« (Mt 27, 27-29), il re glorioso escatologico del mondo (Mt 25, 31-46) diventa, in effetti, il fratello dei più vituperati e il più piccolo tra gli uomini. $\gg$ I crocifissi... diventano il tempio della presenza di Cristo. È una nuova percezione del Dio-con-noi $\left(\right.$ cf. 1, 23; 28, 20). $\ll^{55} \gg$ La novità di Mt 25 consiste

${ }^{49}$ Per esempio U. LUZ, Vangelo di Matteo, III, 659-661; J. RADERMAKERS, Lettura pastorale del Vangelo di Matteo, Bologna, 2001, 314; S. FAUSTI, Una comunità legge il Vangelo di Matteo, 504.

${ }^{50}$ Cf. J. A. T. ROBINSON, The »Parable $\ll$ of the Sheep and the Goats, in: New Testament Studies 2(1956)4, 231.

${ }^{51}$ Così E. FAHARIAN, Relire Matthieu 25, 31-46, in: Gregorianum 72(1991)3, 440-441.

${ }^{52}$ A. MELLO, Evangelo secondo Matteo, 439-440; J. DANIEL - S. J. HARRINGTON, Il Vangelo di Matteo, 319.

${ }^{53}$ Cf. R. HURLEY, Le lecteur et les dans le jugement dernier de Matthieu, in: Sémiotique et Bible 101(2001), 25.

${ }^{54}$ A. FUMAGALLI, Gesù crocifisso, straniero fino alla fine dei tempi. Una lettura di Mt 25, 31-46 in chiave comunicativa, Frankfurt am Main, 2000, 222.

${ }_{55}^{5}$ M. GRILLI, Il discorso della montagna, 76. 
non solo nell'imitatio Dei, ma soprattutto nel definire una nuova percezione della realtà. $\ll^{56}$

La potenza del Figlio dell'uomo glorioso che alla fine dei tempi verrà a giudicare ogni uomo e ogni donna sulla base del loro atteggiamento verso i più piccoli, più bisognosi, più vulnerabili, più indifesi ... fra gli uomini, si realizza nella sua piena identificazione con loro e nella sua continua presenza nei crocifissi di ogni tempo. Questa identificazione e presenza del Figlio dell'uomo nei crocifissi è un serio invito ai discepoli di ogni tempo a una nuova percezione del Dio-con-noi e del suo potere che si trasforma nella responsabilità per i suoi più piccoli fratelli. I discepoli di ogni tempo sono invitati a riconoscere il volto del Figlio dell'uomo nel volto degli affamati, assetati, malati, nudi, forestieri, carcerati ... nella profonda conoscenza che nel personale incontro con lui saranno giudicati sulla base di un solo criterio, l'amore, e che la vera potenza è il servizio ai più piccoli fratelli e sorelle.

Se il re-giudice glorioso ha investito la sua potenza e la sua autorità nella difesa dei più piccoli, e se la loro causa nel giudizio diventa la sua, questo significa che per i suoi discepoli non c'è altro modo di vivere il potere se non quello dell'amore responsabile, gratuito e incondizionato. Non si tratta soltanto di un programma etico o missionario in favore dei poveri, e neppure di un semplice imitatio Dei, ma della dignità del Figlio di Dio intrinsecamente collegata con la dignità dei più piccoli, ai quali i discepoli sono chiamati a servire nell'amore, identificandosi personalmente con il loro destino, nella coscienza che il presente è il tempo dell'agire responsabile. E tuttavia, come giustamente afferma M. Grilli

$\gg$ sarebbe un fraintendimento di Matteo comprendere il giudizio ultimo, contenuto nel cap. 25, come l'affermazione di un cristianesimo definito essenzialmente da un'etica filantropica. Non si tratta di un'etica, anzitutto, ma di una teologia in chiave pragmatica, perché indica ai lettori la strada dell'incontro con Cristo e, ultimamente, con Dio stesso. Cambia la stessa percezione di Dio, includendo a riconoscere la sua presenza non nello spazio e nel tempo sacro, ma nella storia. È certo un elemento di notevole interesse teologico che Matteo concentri qui la presenza di Dio: non nel tempio e nemmeno soltanto nei rappresentanti che parlano a nome di Gesù (cf. 28, 20), ma in chi ha fame, è nudo, è carcerato, ecc. Al posto dell'interesse dei diritti della Chiesa, Matteo pone qui, come criterio ultimo di giudizio, il diritto dell'uomo, di ogni uomo. $\ll^{57}$

\footnotetext{
${ }^{56}$ Ibid., 75 .

${ }^{57}$ Ibid., 76.
} 


\section{Conclusione}

Ritornando all'inizio della nostra indagine sul potere e sull'autorità nella Chiesa alla luce dell'exousia di Gesù, il Figlio dell'uomo a cui è stato dato ogni potere in cielo e in terra, abbiamo ritrovato il vero volto del potere e il senso autentico del servizio della Chiesa. All'interno della comunità credente il modo di vivere il potere e l'autorità non si fonda sui »principi delle nazioni $\ll$ e dei loro $\gg$ grandi $\ll$, ma sul potere del Figlio dell'uomo, che non è venuto a essere servito, ma a servire e dare la vita per la salvezza di tutti. Si tratta dunque del potere che non si fonda sulla logica della sopraffazione, ma del servizio fedele a Dio e solidale con gli uomini secondo il modello del Figlio dell'uomo crocifisso che dona la vita. Lui è $\gg$ il modello e il punto di riferimento per il gruppo dei responsabili della comunità ecclesiale, chiamati a rivivere l'esperienza evangelica che si caratterizza come processo di liberazione umana, di cui essi sono il nucleo e la garanzia. $\ll^{58}$ Non si tratta di un potere come quello che hanno gli uomini che lo esercitano e del quale abusano, ma del potere che non opprime, non domina, non sfrutta e non distrugge l'altro, bensì lo libera e che per ciò è anche esposto al sospetto d'impotenza. ${ }^{59} \gg$ Il modello decisivo di ogni autorità è la vita di Gesù, il Figlio dell'uomo. $\ll^{60}$

L'exousia di Gesù è la potenza che guarisce gli uomini dalle loro infermità facendosi solidale con loro, potenza che scaccia i demoni e perdona i peccati. Il potere di Dio e del Figlio suo è dunque un potere a servizio dell'uomo (cf. Mt 7, 29; 9, 6.8; 10, 1; $21,23.24 .27$ ) e proprio in esso i discepoli di ogni tempo comprendono sé stessi e la loro missione. $\gg$ L'autoreferenzialità che ponesse il potere della chiesa all'origine, al centro o come fine della missione, sarebbe un grave fraintendimento. Non è la chiesa a essere centro e fine, ma l'autorità di Dio, di cui Gesù è dispensatore. $\ll^{61}$ Questo ci mostra chiaramente che $\gg$ non c'è autorità nella Chiesa, se non è una autorità che serve l'uomo; l'autorità nella Chiesa non può che essere a servizio. $\ll^{62}$

Il potere e l'autorità, intesi in questo modo, ci invitano a cambiare la falsa immagine di Dio che anche noi, come i discepoli di Gesù, spesso ci facciamo rifiutando la croce - il luogo epifanico per eccellenza. Luogo dove si infrangono tutte le immagini di Dio, di potere e di autorità. Anche noi come i due figli di Zebedèo e come i discepoli di ogni tempo, troviamo scandalosa e rigettiamo la morte di Gesù sulla

\footnotetext{
58 S. GRASSO, Il Vangelo di Matteo, 598.

${ }^{59}$ U. LUZ, Vangelo di Matteo, IV, Brescia, 2014, 503.

${ }^{60}$ F. LENTZEN-DEIS, Comentario ad Evangelio di Marcos. Modelo de nueva evangelización, Estella, 1998, 325.

${ }^{61}$ M. GRILLI, Parola di Dio e linguaggio umano, 544.

${ }^{62}$ M. GRILLI, Scriba dell'Antico e del Nuovo, 113.
} 
croce. Ma proprio essa come giustamente afferma M. Grilli, ci »obbliga a mettere in discussione le immagini usuali: non solo quelle del Dio potente e fustigatore dei malvagi, ma anche quelle determinate da una visione idolatrica, costruita a proprio uso e consumo. La vera e unica immagine di Dio è l'uomo (Gen 1, 26-27) e, per i cristiani, l'uomo crocifisso (Mc 15, 24ss) ${ }_{*}{ }^{63}$ Questo ci porta a testimoniare, dentro e fuori le nostre comunità, un altro compasso, diverso dai costruttori di questo mondo, il compasso del servizio e del dono di sé rivelato nel Figlio dell'uomo pietra che i costruttori hanno riscattato, ma che è diventata la pietra d'angolo (cf. Mt 21, 42). ${ }^{64} \mathrm{Nel}$ vivere il potere e l'autorità dobbiamo dunque chiederci quale immagine di Dio viviamo e testimoniamo: l'immagine di un Dio potente che rende l'altro schiavo, che lo domina, distrugge e uccide, oppure quella di un Dio potente che serve l'uomo, che lo ama, lo libera e lo fa vivere? Nella ricerca di una risposta autentica e vera ci sembrano di grande aiuto le seguenti riflessioni di J.-M. Ploux:

\Ciò che permette di dire che una rappresentazione di Dio è giusta è che essa serve la vita dell'uomo e la sua umanità. Ed è questa una prima regola a cui attendersi: ogni rappresentazione di Dio che va contro l'uomo, contro la sua vita, contro la sua umanità, che lo sminuisce o lo distrugge è una falsa rappresentazione di Dio. Oppure, ma è la stessa cosa, ogni modo di vivere la religione che porta a disprezzare un uomo, a ridurlo a oggetto o a ucciderlo, nel suo corpo, o nella sua mente, rimanda a un falso Dio. Meglio essere ateo e servire la dimensione umana dell'uomo che essere un religioso posseduto da una rappresentazione di Dio che deforma lo sguardo sull'uomo e che semina la morte. Un Dio degno dell'uomo, un Dio per l'uomo, non può essere altro che un Dio che aiuta l'uomo a diventare più umano e che lo libera da ciò che, dentro di lui o fuori di lui, lo disumanizza. $\ll^{65}$

\footnotetext{
${ }^{63}$ M. GRILLI, Il rantolo di un morente o le doglie di una partoriente? La morte di Gesù nella tradizione sinottica e in Giovanni, in: S. ZENI - C. CURZEL, ed., La speranza della croce, Bologna, 2017, 58 .

${ }^{64}$ Cf. M. GRILLI, L'impotenza che salva, 139.

${ }^{65}$ J.-M. PLOUX, Dio non è quel che credi, Magnano, 2010, 13.
} 


\section{SLUŽENJE JE MOĆ \\ BIBLIJSKO-EVANĐEOSKA I TEOLOŠKA DATOST}

\section{Silvana FUŽINATO*}

Sažetak: Promišljajući o služenju kao načinu življenja moći, potrebno se vratiti Svetomu pismu, posebice Evandelju po Mateju koje prikazuje Isusa kao Sina Čovječjega kojemu je dana sva vlast na nebu i na zemlji (usp. Mt 28, 16-20). U prvom dijelu rada promišljat ćemo o Isusovoj moći ozdravljenja, objavljenoj na poseban i eksplicitan način u Mesijinim djelima (Mt 8 - 9). Moć darivanja života koja proizlazi iz križa tema je drugoga dijela, posvećena hodu Sina Čovječjega prema križu (Mt 20, 17-28). Moć kao odgovornost treća je temeljna značajka Isusove moći objavljene u izvješću o posljednjem sudu Sina Čovječjega (Mt 25, 31-46). Vlast Sina Čovječjega, objavljena u vlasti poučavanja, opraštanja grijeha, ozdravljenja i izgonu zloduha, vlast je u službi čovjeka $i$ njegova spasenja.

Ključne riječi: moć, autoritet, Sin čovječji, služenje, spasenje, Matej.

\footnotetext{
* Doc. dr. sc. Silvana Fužinato, Katolički bogoslovni fakultet u Đakovu Sveučilišta J. J. Strossmayera u Osijeku, P. Preradovića 17, p.p. 54, 31400 Đakovo, Republika Hrvatska, fuzinatosilvana@gmail.com
} 